\title{
Die Megastudien 2008
}

Chefredakteur Dr. med. Dirk Einecke

\section{PLÄTTCHENHEMMER UND AT ${ }_{1}$-BLOCKER}

\section{Was ist optimaler Schlaganfallschutz?}

Was ist die beste Strategie in der Schlaganfall-Sekundärprävention? Untersucht wird dies bei 20000 Patienten. Die Kontrahenten: Hier ASS plus Dipyridamol, dort Clopidogrel. Außerdem wird geprüft, ob ein zusätzliches Sartan, in diesem Fall Telmisartan, die Prognose weiter verbessert. Ergebnisse der PROFESS-Studie im Mai.

\section{HOHES KARDIOVASKULÄRES RISIKO}

\section{$\mathrm{AT}_{1}$-Blocker doch besser als ACE-Hemmer?}

Die HOPE-Studie zeigte, dass kardiovaskuläre Risikopatienten deutlich von einem ACE-Hemmer profitieren: $26 \%$ weniger kardiovaskuläre Mortalität, $20 \%$ weniger Herzinfarkte und 32\% weniger Schlaganfälle als unter Plazebo. In ONTARGET tritt der $\mathrm{AT}_{1}$-Blocker Telmisartan im direkten Vergleich gegen Ramipril an eine dritte Behandlungsgruppe erhält die Kombination. Die Ergebnisse der 5,5-jährigen Studie mit 28000 Patienten werden im März erwartet. Im September wird dann TRANSCEND nachgereicht: Hier werden 5000 Patienten mit dem Sartan behandelt, die den ACE-Hemmer nicht vertragen.

\section{CHOLESTERIN-ABSORPTIONSHEMMER}

\section{Vorteilhaft bei Aortenstenose?}

Was Statine können, ist rauf und runter untersucht worden. Was aber bringt die Kombination Statin plus Ezetimib? 2008 wird es die Ergebnisse der ersten Studien geben: ENHANCE untersucht bei Patienten mit Hypercholesterinämie, ob Ezetimib plus Statin die Atherosklerose besser bremst als ein Statin allein. SEAS prüft, ob Ezetimib plus Statin die Prognose bei Aortenstenose verbessert. Ergebnisse im März und November.

\section{NEUES ANTIARRHYTHMIKUM}

\section{Bessere Prognose bei Vorhofflimmern?}

Die antiarrhythmische Wirkung von Amiodaron ohne die unerwünschten thyreoidalen Effekte: Das erhofft man sich von dem Wirkstoff Dronedaron, der chemisch ähnlich ist, aber kein lod enthält. In großen klinischen Studien hat er bereits bewiesen, dass er Episoden von Vorhofflimmern oder -flattern reduzieren kann. Ob dadurch auch die Morbidität und Mortalität gesenkt werden, sollen die Ergebnisse der ATHENA-Studie (4300 Patienten) in diesem Jahr zeigen.

\section{KHK MIT PUMPSCHWÄCHE}

\section{Mit If-Kanal-Hemmer wirksamer behandelbar?}

Für Patienten mit stabiler Angina pectoris, die nicht mit Betablockern behandelt werden können, ist der If-Kanal-Hemmer Ivabradin eine Alternative. Er führt zu einer Absenkung der Herzfrequenz - ohne andere kardiale Effekte zu haben - und verbessert so die Koronardurchblutung. Ob das Medikament bei KHK-Patienten mit linksventrikulärer Dysfunktion zusätzlich zu einer optimalen Therapie gegeben auch die kardiovaskuläre Morbidität und Mortalität reduzieren kann, wird die BEAUTIFUL-Studie mit 10 ooo Teilnehmern zeigen. Ergebnisse im September.

\section{Zitat der Woche}

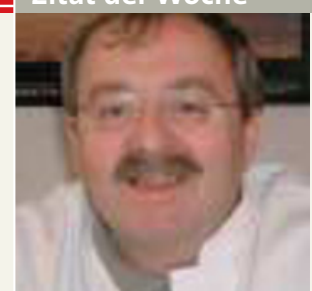

"Mit Metaanalysen ist es wie mit der Bouillabaisse: Wenn ein Fisch stinkt, wird die ganze Suppe schlecht."

Prof. Dr. med. W. Motz, Karlsburg, beim Kongress „Der herzkranke Diabetiker“ am 7. Dezember 2007 in Berlin

\section{DIASTOLISCHE HERZINSUFFIZIENZ}

\section{Was bringt ein Sartan?}

Für Patienten mit Herzinsuffizienz bei erhaltener systolischer Funktion gibt es bisher keine evidenzbasierten Therapieempfehlungen. Der antihypertensiven Therapie wird aber besondere Bedeutung beigemessen. 2008 wird es erstmals gute Daten geben, wie man diesen Patienten helfen kann: Die I-PRESERVE-Studie prüft bei über 4000 Patienten, ob ein $\mathrm{AT}_{1}$-Antagonist (hier: Irbesartan) die kardiovaskuläre Morbidität und Mortalität vermindert.

\section{MEDIKAMENTÖSE THERAPIE DER COPD}

\section{Kann sie die Lungenfunktion erhalten?}

Wenn COPD-Patienten mit dem Rauchen aufhören, können sie damit die Progression ihrer Erkrankung verlangsamen. Für Medikamente wurde ein solcher Effekt bisher nicht nachgewiesen. Das ist das Ziel der UPLIFT-Studie mit Tiotropium, deren Ergebnisse 2008 erwartet werden. Bei knapp 6000 COPD-Patienten wird über einen Zeitrum von vier Jahren untersucht, ob die $\mathrm{FEV}_{1}$ unter dem lang wirksamen Anticholinergikum langsamer abfällt als unter Placebo.

\section{BEI MARCUMAR-KONTRAINDIKATIONEN}

\section{Welcher Plättchenhemmer bei Vorhofflimmern?}

Ein orales Antikoagulanz schützt Patienten mit Vorhofflimmern deutlich wirksamer vor vaskulären Komplikationen als die Therapie mit ASS und Clopidogrel, so das Ergebnis der ACTIVE-W-Studie. Allerdings gibt es Patienten, für die die orale Antikoagulation nicht infrage kommt. Ob diese dann mit ASS allein oder mit ASS plus Clopidogrel besser behandelt sind, wird die Studie ACTIVE A in diesem Jahr zeigen. Im Studienarm ACTIVE I wird außerdem geprüft, ob Irbesartan zusätzlich zur üblichen Blutdrucktherapie den vaskulären Schutz von Vorhofflimmernpatienten weiter verbessern kann. 